\title{
CONSENTIMIENTO INFORMADO EN ADOLESCENTES PARA TOMAR DECISIONES EN TEMAS RELACIONADOS CON SU SALUD, SALUD SEXUAL Y SALUD REPRODUCTIVA
}

\author{
ANA CRISTINA VERA SÁNCHEZ ${ }^{1}$
}

\section{RESUMEN}

Este articulo pretende generar una reflexión legal, desde el marco de los Derechos Humanos y el paradigma de protección integral de los derechos de los niños, niñas y adolescentes sobre el tema del consentimiento informado en adolescentes, y su relación con la patria potestad y con los deberes y obligaciones de los Estados, en base al análisis y aplicación de los principios de bienestar superior y autonomía progresiva.

\section{PALABRAS CLAVE:}

Consentimiento informado, adolescentes, salud sexual, salud reproductiva, bienestar superior y autonomía Progresiva

1 Funcionaria del centro de Apoyo y Protección de los Derechos Humanos SURKUNA 


\begin{abstract}
This article aims to create a legal reflection, from the framework of Human Rights and the paradigm of comprehensive protection of the rights of children and adolescents on the issue of informed consent in adolescents, and their relationship to parental authority and the duties and obligations of States, based on the analysis and application of the principles of higher welfare and progressive autonomy.
\end{abstract}

\title{
KEY WORDS:
}

Informed consent, adolescents, sexual health, reproductive health, superior wellness and progressive autonomy 


\section{INTRODUCCIÓN}

En la actualidad en Ecuador, uno de los debates más importantes en materia de Derechos Humanos de los y las adolescentes es su capacidad para tomar decisiones voluntarias, autónomas, informadas sobre sus derechos relacionados con su salud, vida sexual y vida reproductiva, frente a su derecho a la protección por parte de sus padres y del Estado, que se cristalizan en la patria potestad y en las políticas públicas dirigidas a este sector poblacional.

Este es un debate, que en nuestro país, toma mayor importancia, en el contexto actual, en que las políticas públicas sobre salud sexual y salud reproductiva para adolescentes parten de un paradigma que desconoce a los y las adolescentes como sujetos de derecho y los plantea como sujetos de control$^{2}$; en que las propuestas

2 En diciembre del 2014, el Presidente Correa mediante Decreto Ejecutivo 491 del 12 de diciembre del 2014, transfiere a la Presidencia las funciones de la Estrategia de Planificación Familiar y Prevención del Embarazo en Adolescentes ENIPLA, y la pone bajo control de Mónica Hernández, asesora presidencial con fuertes nexos con grupos religiosos católicos altamente conservadores. Bajo el control de Hernández ENIPLA es transformado en "Plan Nacional de Fortalecimiento de la Familia" más conocido como Plan Familia, una estrategia centrada en la promoción de valores en detrimento de los derechos, el fortalecimiento de la familia, y la educación en abstinencia, que de acuerdo a el borrador de la misma pretende: "lograr un giro significativo en el patrón de comportamiento en adolescentes y jóvenes respecto a la vivencia de la afectividad y la sexualidad, a través del complemento de las políticas públicas en la temática y el fortalecimiento del rol protagónico de la familia" centrando su accionar en la educación sexual en "valores, de responsabilidad y familia" (Hernández, Mónica, Versión Borrador Plan Nacional de Fortalecimiento de la Familia., 2014) 
legales de códigos intergeneracionales como el llamado "código por ciclo de vida"3 resultan fuertemente regresivas en materia de autonomía progresiva y bienestar superior de niños niñas y adolescentes ${ }^{4}$ y en los que cada día existen más barreras para que jóvenes y adolescentes puedan ejercer sus derechos y acceder a servicios de salud sexual y salud reproductiva ${ }^{5}$.

Es entonces que se vuelve de transcendental importancia generar una reflexión legal, desde el marco de los Derechos Humanos y el paradigma de protección integral de los derechos de los niños, niñas y adolescentes sobre el tema del consentimiento informado en adolescentes, y su relación con la patria potestad y con los deberes y obligaciones de los Estados.

Este artículo pretende aportar para esta reflexión crítica, partiendo del análisis del marco jurídico nacional e internacional

http:/ /www.planfamilia.gob.ec/

3 https://www.dropbox.com/s/4nbf76sp9h7yika/Proyecto $\% 20$ de\%20Ley \%20del\%20C\%C3\%B3digo \%20de \%20Ciclo\%20de \%20 Vida\%20T.pdf?dl=0

4 El 21 de marzo del 2016, la asambleísta de Alianza País, Zobeyda Gudiño, presento la propuesta de Código por ciclo de vida, la misma que fue fuertemente criticada por varios sectores y archivada al ser catalogada como la forma de transformar códigos y comportamientos morales altamente conservadores en ley. http://www.planv.com.ec/historias/sociedad/codigociclo-vida-manual-correista-regular-la-vida

5 Actualmente las barreras y brechas que adolescentes y jóvenes enfrentan para acceder a servicios de salud, salud sexual y salud reproductiva generan que los mismos no puedan ejercer sus derechos. Para profundizar al respecto de esto puede leer la investigación: Nuestros Derechos, Nuestras Voces, realizada en el año 2016 por el Centro de Apoyo y Protección de Derechos Humanos SURKUNA. 
sobre derechos de los y las adolescentes, de recomendaciones de comités internacionales y relatores, y de sentencias de cortes internacionales y de otros países sobre esta problemática.

Es fundamental aclararque para el presente artículo distinguiremos entre niños, niñas y adolescentes, como dos situaciones jurídicas diferenciadas. Abordando para el presente análisis únicamente la situación de adolescentes.

Entenderemos a la adolescencia como una edad comprendida entre 10 y 19 años, dónde se evidencian cambios biológicos, psicológicos y sociales en la persona, que marcan la transición de los patrones de la niñez a la adultez y consolidación de su independencia (UNICEF, 2011, Estado Mundial de la Infancia $2011,8-10)^{6}$. Y que por fines prácticos, las reflexiones que se generan en el presente articulo son válidas únicamente para las y los adolescentes considerados como menores de edad, es decir menores de 18 años ${ }^{7}$.

6 Existe una discusión sobre cuáles son las edades en las que se encuentra comprendida esta etapa denominada como adolescencia, de acuerdo a UNICEF las edades que comprende van de 10 a 19 años, definición que es concordante con la establecida por parte de nuestro MSP cuando aborda el tema de la adolescencia como un ciclo de vida. No obstante nuestro código de la niñez y la adolescencia en su artículo 4, establece que se entiende como adolescente a toda persona comprendida entre los 12 y 18 años, y la Convención por los Derechos de los Niños, dentro de la cual se encuentran comprendidos los derechos de las y los adolescentes, establece como estándar para efectos de la protección que brinda a los 18 años.

7 La Convención sobre los Derechos de los Niños, constituye el marco normativo más relevante en cuanto al estándar de la minoría de edad. La misma establece en su artículo 1, que para efectos de la protección la minoría de edad va hasta los 18 años. Estándar que es 
Esto por cuanto es alrededor de los derechos de los y las adolescentes menores de edad donde se generan los dilemas sobre su capacidad legal de tomar decisiones, debido a que la definición internacional de minoría de edad, tiene un doble propósito, establecer una protección especial para los menores de modo que sus derechos primen sobre los de otros y sean especialmente protegidos por el Estado, la sociedad y la familia, e imponer restricciones al ejercicio de los derechos y de las obligaciones por parte de los menores hasta que se presuma que tienen suficiente madurez para comprender sus decisiones y asumir las consecuencias de sus actos.

Este trabajo está dividido en varios acápites, en el primero se abordara brevemente el tema de derechos de los niños, niñas y adolescentes, la titularidad de los mismos y principios para su ejercicio; en el segundo se analizara a partir de estos conceptos el consentimiento informado en adolescentes en temas relativos a su salud sexual y su salud reproductiva.

\section{DERECHOS DE LOS Y LAS ADOLESCENTES, TITULARIDAD Y PRINCIPIOS PARA SU EJERCICIO}

Existen varios paradigmas sobre derechos de niños, niñas y adolescentes. Sin embargo, en la actualidad la más aceptada es la doctrina de protección integral (SIMON, Farith, 2008, Derechos de la Niñez y Adolescencia: De la Convención sobre los del Niño a las Legislaciones Integrales, 63) de acuerdo a la cual los mismos son titulares de derechos en varias dimensiones: política, económica, social, cultural, sexual, reproductiva, y de derechos

coherente con la mayoría de legislaciones de Latinoamérica y con la legislación del Ecuador. 
colectivos (Aguilar Carvallo, Gonzalo, 2008, "El principio del interés superior del niño y la Corte Interamericana de Derechos Humanos", 229), que ejercen de acuerdo a su madurez y desarrollo progresivo.

La existencia de Derechos Humanos específicos de niños, niñas y adolescentes, desde esta perspectiva, no genera regímenes excepcionales sino que interpreta y adapta el régimen general de los Derechos Humanos a las particularidades de estos rangos etarios, generando un sistema jurídico de protección de niños, niñas y adolescentes en tanto titulares de derechos tanto en el ámbito internacional como en el local (Agullar Carvallo, Gonzalo, 228-230).

El principal instrumento internacional en materia de Derechos de los niños, niñas y adolescentes, es la Convención sobre los Derechos del niño del 20 de noviembre de 1989, la misma que ha sido firmada y ratificada por 192 Estados (incluido Ecuador), lo cual demuestra su amplio grado de reconocimiento y aceptación a nivel mundial. Esta convención es de obligatorio cumplimiento para el país y forma parte del bloque de constitucionalidad (García Falconí, José, "Tratados Internacionales de Derechos Humanos: Diferenciación con otros tratados” 2014).

En cuanto a legislación interna, la constitución de la República constituye un instrumento fundamental en materia de derechos Humanos de niños, niñas y adolescentes, al igual que el Código de la Niñez y la adolescencia vigente en la actualidad. Los mismos que reiteran lo establecido en la Convención de Derechos del Niño, reconociendo la titularidad de derechos de niños, niñas y adolescentes; la obligación de protección especial a los mismos por parte del Estado, la sociedad y la familia; y el 
ejercicio progresivo de estos derechos de acuerdo a su madurez y desarrollo (SIMON, Farith, 63)

De acuerdo a todos estos instrumentos legales los y las adolescentes menores de edad son titulares de derechos, cuyo ejercicio debe regirse por dos principios fundamentales, el principio de interés superior y el de autonomía progresiva.

El principio de interés superior, es cardinal en materia de derechos de los niños, niñas y adolescentes, el mismo ha sido reconocido a nivel internacional y nacional como un principio general de derechos $^{8}$ y como un principio integrante del sistema jurídico de protección de los derechos de niños, niñas y adolescentes (Aguilar Carvallo, Gonzalo, 226-227). El mismo se encuentra establecido en el artículo 3 de la Convención por los Derechos del niño de forma general y en los artículos 9.1, 9.3, art. 18.1 y art. 20.1. de la misma Convención en forma específica, y tiene su correlato en nuestra legislación en el artículo 44 de la Constitución de la República y el artículo 11 del Código de la Niñez y la Adolescencia.

Este principio hace referencia a los niños, niñas y adolescentes como sujetos de derechos (SIMON, Farith, 319) y establece que sus derechos prevalecen sobre los de cualquier persona; que los mismos requieren de una protección reforzada del Estado, la sociedad y la familia, por la vulnerabilidad fáctica que implica la minoría de edad (GonzÁlez Vélez, Ana Cristina y Durán, Juanita, 2010, Derechos sexuales y reproductivos de la gente joven, 12) y que las limitaciones que se impongan al ejercicio de derechos por parte de la Corte Internacional de Justicia. 
de niños y adolescentes no pueden causarles daño, desprotegerlos, ni generarles condiciones de mayor vulnerabilidad (GoNZÁLEZ VéleZ, Ana Cristina y DuRán, Juanita, 12)

El principio de bienestar superior del niño en su desarrollo y aplicación plantea que las prerrogativas, derechos y garantías del régimen de protección internacional de Derechos Humanos de los niños, pueden ser aplicadas también en ámbitos privados, siendo posible la utilización y consideración del mismo para analizar las relaciones familiares, y el rol de los padres como garantes de derechos (Aguilar Carvallo, Gonzalo, 235).

El mismo se formula como un mandamiento amplio y por tanto como un concepto jurídico indeterminado, cuya aplicación requiere de la evaluación de la situación fáctica de niños, niñas y adolescentes para determinar la idoneidad de la misma para el ejercicio de sus derechos.

En cuanto a su función, este principio es multidimensional (SIMON, Farith, 79-89 y 319) pues actúa en el desarrollo de derechos, su determinación, su sustentación, su limitación y su aplicación; ya que sirve como principio orientador de las autoridades legislativas, judiciales y administrativas, y como cláusula de prioridad frente a conflictos que requieran ponderación de derechos.

El principio de autonomía progresiva, por su parte, implica que de acuerdo a sus capacidades evolutivas niños, niñas $\mathrm{y}$ adolescentes van ganando autonomía para la toma de decisiones sobre el ejercicio de sus derechos y sobre sus vidas. $\mathrm{Su}$ implementación requiere del establecimiento de un equilibrio entre el reconocimiento de las y los adolescentes como agentes activos en sus propias vidas que tienen derecho a ser respetados 
como ciudadanos, como personas y como titulares de derechos con una autonomía creciente, y el derecho que tienen a diferentes niveles de protección de acuerdo a su grado de vulnerabilidad (GonZÁlez Vélez, Ana Cristina y Durán, Juanita, 12-15).

La clave de este principio es la autonomía, la misma que se desarrolla conforme niños, niñas y adolescentes van creciendo, y adquiriendo competencia para tomar decisiones que afecten sus vidas (SIMON, Farith, 39).

Este principio actúa en dos sentidos, permitiendo y garantizando el ejercicio de derechos por parte de adolescentes cuando existan las condiciones e impidiendo su adultización prematura (SIMON, Farith, 2010, "Garantías de los derechos de la infancia y adolescencia”, 475).

Y tiene como objetivo promover el desarrollo, la competencia y la gradual autonomía de niños, niñas y adolescentes; valorar las contribuciones que ellos y ellas hacen hacia su propia protección; generar de condiciones donde se potencie su participación y la responsabilidad sobre sus decisiones; garantizar su protección especial e impedir injerencias arbitrarias en su vida que puedan convertir su minoría de edad en una condición de vulnerabilidad (GonZÁlez Vélez, Ana Cristina y Durán, Juanita, 18-20).

Este principio se encuentra establecido en el artículo 5 de la Convención de Derechos del Niño y tiene su correlato en el artículo 44 y 347.5 de la Constitución de la República y en el artículo 13 del Código de la Niñez y la adolescencia.

El mismo se complementa con el reconocimiento realizado por parte de la Convención sobre los Derechos del Niño, de que las 
restricciones a los derechos de las y los adolescentes realizadas supuestamente para su protección no pueden causarles daño, ni afectar sus derechos y su bienestar, pues en caso de hacerlo las mismas se vuelven inválidas por vulnerar la protección de éstos, que son el principio de su existencia y constituirse en injerencias arbitrarias. Tesis que se ve respaldada por el artículo 16.1 de la convención que prohíbe las injerencias arbitrarias o ilegales en la vida privada de niños, niñas y adolescentes y el establece el derecho a la protección estatal de las y los menores cuando esto suceda.

Artículo 16 1. Ningún niño será objeto de injerencias arbitrarias o ilegales en su vida privada, su familia, su domicilio o su correspondencia ni de ataques ilegales a su honra y a su reputación. 2. El niño tiene derecho a la protección de la ley contra esas injerencias o ataques. (Convención sobre los Derechos de los niños, 1989)

Todo lo expuesto anteriormente, nos permite generar un escenario para analizar el tema de derechos de niños, niñas y adolescentes, desde el cual si bien existe un ejercicio limitado de los derechos para estos grupos por temas de protección, existen también en contrapartida limitaciones a patria potestad de los padres y madres, en base a los principios de interés superior de los niños, niñas y adolescentes y de autonomía progresiva; con el objetivo de garantizar que no exista un ejercicio desproporcionado de la autoridad que vulnere los derechos de los menores. 


\section{CONSENTIMIENTO INFORMADO EN ADOLESCENTES EN EL MARCO DE LA AUTONOMÍA PROGRESIVA Y BIENESTAR SUPERIOR DE LOS NIÑOS, NIÑAS Y ADOLESCENTES}

El consentimiento Informado, es un derecho de las personas en salud, que se entiende como la expresión autónoma de su voluntad para elegir, autorizar o negar un determinado procedimiento, posteriormente a una adecuado información proporcionada sobre el mismo, sus riesgos, beneficios, alternativas y posibles cursos alternativos (PACHECO, Víctor Manuel, 2015, Consentimiento Informado (fundado), 1).

Su fundamento es el respeto al Derecho Humano a la Libertad, traducido como principio de autonomía y autogestión de las personas de su propia vida de acuerdo a sus valores y deseos (PACHECO, Víctor Manuel, 1).

El consentimiento informado tiene dos componentes:

1. Derecho a recibir información clara, veraz y oportuna sobre todos los aspectos del procedimiento objeto del consentimiento; y,

2. El derecho a decidir de manera autónoma, sin interferencias, violencia o coerción sobre si aceptar o rechazar determinado procedimiento.

En Ecuador en materia de consentimiento informado de las y los adolescentes no existe una normativa clara, razón por la que es fundamental analizar el tema de consentimiento informado en adolescentes para tomar decisiones concernientes a su salud a la luz de los principios de bienestar superior y autonomía progresiva, con el objetivo de garantizar el ejercicio de los derechos de los adolescentes. Teniendo en cuenta que existe una tensión entre la posibilidad de que ellos ejerzan sus derechos de forma autónoma, 
y su derecho a la protección en base a la cual muchas veces sus derechos son limitados por la patria potestad de sus padres y madres.

En este sentido, es importante reflexionar, como generar un equilibrio ente el reconocimiento de los y las adolescentes como agentes activos en sus propias vidas con autonomía creciente, y el derecho que tienen a diferentes niveles de protección de acuerdo a su grado de vulnerabilidad. (Convención sobre los Derechos de los niños, 1989).

Al abordar el tema de consentimiento informado en caso de menores de edad, desde la consideración de la regla del menor maduro o autonomía progresiva y la regla del bienestar superior de niños, niñas y adolescentes, implica el reconocimiento de la madurez de las y los adolescentes menores de forma independiente a su edad, sin que esto restrinja la posibilidad de establecer edades límites para la subrogación de la decisión en determinadas prácticas; y la garantía de que las decisiones de sustitución que se tomen sobre la salud de o la menor en caso de ser necesario no le causen daño.

Como regla general, si el menor es capaz de comprender, intelectual y emocionalmente, el alcance de la intervención, es él quien debe tomar la decisión autónomamente. Si el menor no cuenta con estas condiciones el consentimiento lo debe prestar el representante legal, después de haber escuchado su opinión cuando es mayor de doce años. Si el menor tiene más de dieciséis años o está emancipado, no se puede prestar el consentimiento por representación (GonZÁlez VÉlEZ, Ana Cristina y Durán, Juanita, 18). 
La comprensión de estos conceptos requiere reconocer que los y las niños, niñas y adolescentes van adquiriendo progresivamente condiciones para adoptar decisiones autónomas sobre su vida y ejercer los derechos de los que son titulares. Y que por tanto el ejercicio de la protección paternal o maternal, traducida en la subrogación del consentimiento en salud, solo es legitima cuando efectivamente la precariedad del juicio y discernimiento del niño, niña o adolescentes le hace requerir de la misma como una forma de protección.

Esto implica que las capacidades evolutivas de las y los menores de edad pueden ser entendidas como una forma de limitación al ejercicio de la autoridad parental, en favor del ejercicio de sus derechos de las y los adolescentes de acuerdo con su madurez. Esto se cristaliza en la toma de decisiones sobre su salud y vida aun cuando las mismas contraríen las expectativas o deseos de sus progenitores.

El concepto de menor madura/o es la regla a través de la cual se establece el respeto a las capacidades evolutivas de las y los adolescentes. Según esta regla:

(...) cuando este puede comprender la información que se le brinda y el alcance de los actos, sus riesgos y sus consecuencias, debe ser él quien decida,, partiendo de la constatación empírica según la cual los menores adquieren "madurez moral" mucho antes de ser mayores de edad, independientemente de los años cumplidos, aunque algunos se puedan tardar más que otros. Por consiguiente, dicho enfoque supone que los menores pueden ejercer sus derechos desde el momento que pueden gozarlos (GonzÁlez Vélez, Ana Cristina y Durán, Juanita, 19). 
Tomando en cuenta esto y el principio de bienestar superior del niño, niña y adolescente de acuerdo al cual en cualquier caso la titularidad de derechos de los menores se mantiene sobre las limitaciones que pueden establecerse al ejercicio de los mismos, por lo cual la restricción a los derechos de las y los adolescentes no puede causarles daño, ni afectar sus derechos y su bienestar, en caso de hacerlo se vuelve inválidas por vulnerar la protección de las y los niños, niñas y adolescentes que son el principio de su existencia.

Art. 11.- El interés superior del niño.- El interés superior del niño es un principio que está orientado a satisfacer el ejercicio efectivo del conjunto de los derechos de los niños, niñas y adolescentes; e impone a todas las autoridades administrativas y judiciales y a las instituciones públicas y privadas, el deber de ajustar sus decisiones y acciones para su cumplimiento. Para apreciar el interés superior se considerará la necesidad de mantener un justo equilibrio entre los derechos y deberes de niños, niñas y adolescentes, en la forma que mejor convenga a la realización de sus derechos y garantías. Este principio prevalece sobre el principio de diversidad étnica y cultural. El interés superior del niño es un principio de interpretación de la presente Ley. Nadie podrá invocarlo contra norma expresa y sin escuchar previamente la opinión del niño, niña o adolescente involucrado, que esté en condiciones de expresarla (Código de la Niñez y la adolescencia, 2003)

Artículo 16 1. Ningún niño será objeto de injerencias arbitrarias o ilegales en su vida privada, su familia, su domicilio o su correspondencia ni de ataques ilegales a su honra y a su reputación. 2. El niño tiene derecho a 
la protección de la ley contra esas injerencias o ataques.

(Convención sobre los Derechos de los niños, 1989)

Retomando estos fundamentos en la discusión del consentimiento informado en niños, niñas y adolescentes, queda claro que las y los adolescentes tienen derecho a dar su consentimiento sobre decisiones concernientes a su salud, de acuerdo a su madurez y de forma progresiva y autónoma. Lo cual implica que se debe propiciar que los y las adolescentes menores tomen la decisión y en caso de que realmente no tengan capacidad para hacerlo, la decisión debe ser sustituida, garantizándose siempre que las decisiones de sustitución que se tomen sobre la vida de los y las adolescentes menores no le causen daño.

En este sentido, el Estado tiene una obligación mucho más amplia en materia de garantía de los Derechos de las y los adolescentes, incluido su derecho a decidir sobre su salud, pues por un lado tiene la obligación de garantizar el ejercicio de estos derechos, conforme los reconoce como titulares de los mismos con autonomía creciente y por otra tiene la obligación de ofrecerles protección especial frente a los diversos grados de vulnerabilidad que puedan dificultar el ejercicio de sus derechos, tanto si estos obstáculos derivan de su falta de madurez suficiente o de un intento de injerencia abusiva de sus padres y madres sobre su vida privada. Por lo cual el Estado en algunas ocasiones tiene la obligación de limitar la patria potestad de los padres y madres, de acuerdo al principio de interés superior de los niños, niñas y adolescentes; y su autonomía progresiva. Esto con el objetivo de garantizar que no exista un ejercicio desproporcionado de la autoridad que vulnere los derechos de los y las adolescentes. 
Así, el Estado ecuatoriano debe garantizar que las restricciones a los derechos de las y los adolescentes estén fundamentadas, y que en ningún caso puedan afectar su bienestar.

\section{CONSENTIMIENTO INFORMADO DE LAS Y LOS ADOLESCENTES EN TEMAS DE SALUD SEXUAL Y SALUD REPRODUCTIVA}

El derecho de las y los adolescentes a consentir sobre temas de salud sexual y salud reproductiva debe ser analizado desde una perspectiva amplia, que nos permita por un lado generar una reflexión sobre los Derechos Sexuales y Derechos Reproductivos de las y los adolescentes y su ejercicio; y por otro sobre el consentimiento informado en diversos procesos relacionados con la salud sexual y salud reproductiva de las y los adolescentes.

Paracomenzarestareflexión es necesario plantearquelos Derechos Sexuales y Derechos Reproductivos ${ }^{9}$, han sido reconocidos por la comunidad universal como Derechos Humanos en tratados, convenciones y acuerdos aprobados internacionalmente ${ }^{10}$. Y

9 Estos derechos incluyen: tomar decisiones libres, informadas y voluntarias sobre su sexualidad; no ser discriminadas por orientación sexual o identidad de género; derecho a la confidencialidad de la información en salud y sobre su vida sexual; el acceso a servicios de salud basados en los principios de la bioética, que respete las condiciones intergeneracionales, interculturales, de género, con eficiencia, trato digno, calidad y calidez; la autodeterminación reproductiva; el derecho a la integridad personal física, psíquica, moral y sexual; el derecho a una vida libre de violencia en el ámbito público y privado planteándose la obligación del Estado de adoptar las medidas necesarias para prevenir, eliminar y sancionar toda forma de violencia; y el derecho fundamental de las personas una vida digna.

10 A partir de la Conferencia Internacional de Población y Desarrollo de El Cairo (1994) y su Programa de Acción respectivo que se 
que los mismos, son reconocidos por nuestra Constitución de la República del Ecuador en sus artículos 66 y 32 y por nuestra normativa interna en el Art. 259 de la Ley Orgánica de Salud, como Derechos Humanos Fundamentales e inherentes a la libertad de las personas.

En este sentido y de acuerdo a nuestra Constitución, artículo 11, estos Derechos son de inmediata y directa aplicación sin ningún tipo de discriminación, su contenido no pueda ser restringido y no puede haber regresividad ni en su aplicación ni en su conceptualización. Siendo el rol del Estado respetarlos, proteger su el ejercicio y garantizarlos.

Los y las adolescentes en tanto titulares de derechos, son titulares también de los Derechos Sexuales y Derechos Reproductivos, ya que como lo establece nuestra Constitución, artículo 45, "Las niñas, niños y adolescentes gozarán de los derechos comunes del ser humano, además de los específicos de su edad." (Constitución).

reconoce la existencia de los derechos reproductivos, y acuña el "derecho a alcanzar el nivel más elevado de salud sexual y reproductiva" (Naciones Unidas, 1995) Siendo no obstante, en la Cuarta Conferencia Mundial sobre la Mujer de 1995, donde se establece la garantía del Derecho a la salud y los Derechos Sexuales y Derechos Reproductivos como fundamentales para el ejercicio de los Derechos Humanos y el desarrollo, calificando enfoques en todo proceso de comprensión integral de los Derechos sexuales y derechos reproductivos como DDHH y legitimando también los derechos sexuales (Naciones Unidas, 1996). En esta conferencia se generó el documento más completo producido por una Conferencia de Naciones Unidas con relación a los derechos de las mujeres ya que incorpora lo logrado en conferencias y tratados anteriores. 
Nuestra Constitución además al establecer a este grupo poblacional como prioritario, genera una obligación estatal de garantizar prioritaria sus derechos, y a la vez de vigilar que los mismos no sean vulnerados.

Sin embargo, cuando hacemos referencias a los Derechos Sexuales y Derechos Reproductivos de las y los adolescentes, no debemos olvidar que el ejercicio de los mismos debe estar vinculado a la madurez de los adolescentes, y su bienestar, entendido no desde una lógica adultocéntrica, sino partiendo de un análisis de las condiciones de la o el adolescente y de un diálogo conjunto con él o ella. Siendo que hay ocasiones en que un derecho puede limitarse como una forma legítima de protección y otras en que su limitación implica una efectiva vulneración que no debe ser permitida. Pongamos un ejemplo, negar el acceso de adolescentes a información sobre sexualidad, los expone a tener prácticas sexuales inseguras que pueden generar impactos a largo plazo en sus vidas y por tanto vulneraría sus derechos (JIMBO, Ruth, 2010, Educación sexual versus abstinencia en adolescentes, 6-7), en cambio como contrapartida, permitir la libre asociación sexual de adolescentes menores de 14 años, con adultos generar vulnerabilidad para los primeros pues los expone a formas de manipulación y violencia generadas por la diferencia de edad, y por tanto en este caso es necesario establecer una limitación para este derecho (VALENZuela, Lidia y CASAS, Ester, 2010, "La regulación legal sobre la capaciad de los adolescentes en algunos países de América Latina y su impacto en la salud sexual y reproductiva", 45-47).

Uno de los derechos sexuales y derechos reproductivos de la población adolescentes es el acceso a servicios de salud en estos ámbitos de forma confidencial y prioritaria, garantizando la 
posibilidad de que los mismos puedan consentir determinadas prácticas sin necesidad de autorización de sus madres o padres, desde una perspectiva de derechos humanos de niños, niñas y adolescentes, y que por tanto tome en cuenta los principios de bienestar superior y autonomía progresiva.

En este sentido debemos analizar, que situación garantiza de mejor manera los derechos de las y los adolescentes: a) que puedan acceder a servicios de salud sexual y salud reproductiva sin autorización de sus padres o b) que tengan limitado su acceso a los mismos y sus decisiones en salud estén supeditadas a la opinión de sus padres.

Para indagar en este dilema, debemos comenzar evaluando cuáles son las consecuencias de no acceder a servicios de atención en salud sexual y salud reproductiva por parte de la población adolescente, y cuál es el papel que juegan padres y madres en la educación sexual de sus hijos e hijas y su acceso a servicios de salud en estos ámbitos.

De acuerdoa múltiplesestudios, entrelasprincipalesconsecuencias de la falta de acceso por parte de adolescentes a servicios de salud sexual y salud reproductiva se encuentra: el aumento de los riesgos de prácticas inseguras y sus consecuencias: embarazos adolescentes, ITS incluido el VIH, Abortos inseguros, mortalidad materna, violencia sexual sistemática (CHRISTOFIDES, 2014, Risk factors for unplanned and unwanted teenage pregnancies occurring over two years of follow-up among a cohort of young South African women); la instauración de estilos de vida riesgosos que pueden tener impactos potenciales en la calidad de vida de las personas (Maddaleno, Matilde, Morelo, Paola e Infante, Francisco, 2003, "Salud y desarrollo de adolescentes y jóvenes en 
Latinoamérica y El Caribe: desafios para la próxima década", 133); la restricción de la capacidad de las personas de tomar decisiones reproductivas, lo cual repercute en su incapacidad para planificar sus vidas y cumplir con sus planes de vida (TRIVEDI D, BunN F, Graham M, Wentz R., 2007, Update on review of reviews on teenage pregnancy and parenthood. Centre for Research in Primary and Community Care University of Hertfordshire, 49); la reproducción de la inequidad de género y su mantenimiento por el aumento de las disparidades en educación y trabajo para las mujeres. Si la familia es más numerosa, especialmente si hay más de cinco hijos, la probabilidad de que las niñas permanezcan en la escuela disminuye notablemente (INEC, Censo 2010, 2010) la reproducción de la inequidad social y económica, potenciando el mantenimiento de los círculos de pobreza y de las desigualdades. El embarazo adolescente ha sido asociado con un bajo rendimiento educacional y con otros factores asociados a pobreza (Trivedi D, Bunn F, Graham M, Wentz R., 275291); la interrupción o discontinuación de la educación, muchas adolescentes embarazadas se ven forzadas a abandonar la escuela y con ella sus aspiraciones laborales, bien por la implicación social, la implicación física del embarazo o por expulsión directa por parte del centro. El 59,1\% de mujeres que se embarazaron entre 15 y 24 años interrumpió sus estudios a causa del embarazo, de este porcentaje 44, 3\% no volvió a retomarlos (ENSANUT 2013); y la "maternización" de niñas que niega su derecho a vivir la adolescencia y las somete a maternidades forzadas, afectando su salud integral (Fundación Desafío. Vidas Robadas: entre la omisión y la premeditación, 2015).

Como podemos mirar en toda la evidencia esgrimida, el no acceso a servicios y la limitación de las decisiones de adolescentes sobre su salud sexual y salud reproductiva afecta sus derechos sexuales 
y derechos reproductivos, pero también vulnera otros Derechos Humanos fundamentales de esta población, como lo son su derecho a la salud, a la integridad, a la vida, a la confidencialidad, privacidad, y educación (R.CoOK, B.M.DiCKENS, 2005, El reconocimiento de "la evolución de las facultades" de los adolescentes para tomar decisiones en cuanto a su salud reproductiva, 20-21). Generando este no acceso que la minoría de edad se convierta en un factor de inequidad, vulnerabilidad y desprotección.

En ese sentido, se debe reconocer que la minoría de edad no puede convertirse en un factor de vulnerabilidad ni se puede utilizar para permitir o causar daños, como la desprotección de los derechos a la salud y la integridad de los menores, lo cual sucede cuando se niega su posibilidad de decidir en relación con su sexualidad y su reproducción (GonzÁlez VéLEZ, Ana Cristina y Durán, Juanita, 12).

Razón por la que puede afirmarse que negar el acceso de adolescentes a estos derechos constituye una injerencia arbitraria en su vida. Siendo que el acceso a servicios de salud y la posibilidad de tomar decisiones sobre estos aspectos de su vida, constituyen medios de protección de sus derechos y de su integridad (R.COOK, B.M.DiCKENS, 20-21).

El segundo factor de análisis para clarificar este dilema es el papel que juegan padres y madres en la educación sexual de sus hijos e hijas y en su acceso a servicios de salud sexual y salud reproductiva.

De acuerdo a estadísticas, en el Ecuador el medio por el cuál las personas se enteran de temas relacionados con sexualidad 
es la televisión (20\%). Sin embargo, no existen datos sobre el tipo de mensajes que los y las adolescentes adquieren a través de este medio. ${ }^{11}$ Siendo otras fuentes importantes de información la escuela o colegio y estando en un lugar muy inferior en cuanto a la información sobre estos temas la familia (FREIRE W. B., Ramírez M.J., Belmont P., Mendieta M.J., Silva K.M., ROMERO N et al., 2013, Encuesta nacional de salud y nutrición 2011-2012, 272) ${ }^{12}$, hecho que se debe a la falta de información en padres y madres para hablar sobre su sexualidad con sus hijos e hijas, a sus sentimientos de vergüenza y actitudes de silencio y desaprobación (UNESCO, 2010, Orientaciones Técnicas Internacionales sobre Educación en Sexualidad, 11).

Estudios muestran que incluso en los casos donde padres y madres juegan un rol importante en la educación sexual de sus hijos e hijas, la información que estos tienen no siempre es completa, basada en evidencia y adecuada a la edad, lo cual puede influir en que las y los adolescentes vivan su sexualidad a partir de creencias y mitos perjudiciales para el ejercicio de la misma (UNIFEC y Plan Internacional, 2014, Vivencias y relatos sobre

11 Se conoce, no obstante, que en EEUU los y las adolescentes son receptores de aproximadamente 14.000 referencias anuales a temas sexuales. Sin embargo, solo 165 de estos mensajes se refieren a prácticas sexuales responsables, uso de métodos anticonceptivos, abstinencia y el riesgo de embarazos no planeados o ITS. (UNFPA, Equipo de Apoyo Técnico de América Latina y el Caribe, s/f)

12 De acuerdo a las estadísticas el 20\% de las mujeres entre 15 y 24 años de edad no ha recibido información sobre pubertad; el 17,2\% no han recibido información sobre los aparatos reproductores, el $6,8 \%$ no han recibido información sobre menstruación; el 13,9\% no han recibido información sobre relaciones sexuales; el 15,3\% no ha recibido información sobre métodos anticonceptivos; $13,7 \%$ no ha recibido información sobre VIH e ITS; y el 47\% no ha recibido información sobre anticoncepción oral de emergencia. 
el embarazo en adolescentes. Una aproximación a los factores culturales, sociales y emocionales a partir de un estudio en seis países de la región. Informe Final, 42 y 68).

En cuanto a acceso a servicios de salud sexual y salud reproductiva, la evidencia muestra que padres y madres suelen tener una fuerte resistencia de llevar a sus hijos e hijas a los mismos por creencias erróneas sobre su papel para promover relaciones sexuales tempranas en adolescentes (UNICEF y Plan Internacional, 39 y 43) y que las y los adolescentes suelen tener sentimientos de vergüenza y miedo de ir a servicios de salud con sus padres y madres pues esto generalmente responde a lógicas de control de su vida sexual y no de protección. (SURKUNA, 2016, 36 y 37). Esta es la causa por la cual en Ecuador el grupo de adolescentes entre 15 y 19 años es el grupo poblacional que ha asistido a la menor cantidad de consultas por morbilidad en el país y por tanto el más expuesto a los riesgos antes descritos (MSP).

El análisis de esta segunda variable, nos permite afirmar que limitar el acceso de adolescentes a servicios de salud sexual y salud reproductiva al no permitirles tomar decisiones sobre estos aspectos de su vida, repercute en su efectivo no acceso a los mismos y trae como consecuencias todos los impactos negativos antes mencionados. En este sentido, esta limitación también vulneraría los derechos de las y los adolescentes, y se constituirá por tanto en un injerencia arbitraria en su vida.

Esto implica que al negarse el acceso de adolescentes a servicios de salud sexual y/o salud reproductiva o reducirse este acceso al no permitirles tomar decisiones sobre su salud, su sexualidad y su reproducción, se vulneran todos estos derechos. Constituyendo estas restricciones una 
injerencia arbitraria en su vida privada, que vulnera su bienestar superior. Todas las limitaciones para decidir de las/los menores de edad deben interpretarse siempre restrictivamente, respetando lo más posible su autonomía y trasladando, a quien se opone a que decida de manera autónoma o considera que carece de condiciones hacerlo, la carga de probarlo.21 También deben interpretarse como una medida de protección y no como una limitación, por lo que no pueden ser usadas para desproteger a los menores (GARCíA, Diego, y otros, 2001, Toma de decisiones en el paciente menor de edad. Instituto de Bioética. Fundación Ciencias de la Salud, 181).

En este sentido se puede concluir que es fundamental para la garantía de los derechos de las y los adolescentes, y de su bienestar superior que se les permita acceder a servicios de salud sexual y salud reproductiva y tomar decisiones sobre estos aspectos de sus vidas.

Otro dilema que surge en el marco de esta discusión, es si permitir el acceso de adolescentes a servicios de salud sin autorización de sus padres y madres atenta contra la patria potestad de los mismos y de otros derechos que tienen en su calidad de progenitores.

Para analizar esto debemos empezar por recordar que la patria potestad no puede vulnerar los derechos de las y los adolescentes, pues en ese caso violaría el principio de su existencia, que es la protección de estos. Razón por la cual no puede aducirse vulneración de esta para evitar que adolescentes accedan a servicios de salud sexual y salud reproductiva de forma integral, a menos que se pruebe que la restricción de este acceso es efectivamente un factor de protección del bienestar superior del 
menor, lo que sucede en muy pocos casos, siendo la regla general como se demostró anteriormente que estas restricciones lesionan sus derechos y los ponen en situaciones de indefensión.

Al respecto,el caso "Gillik vs West Norfolk \& Wisbech Area Health Authority" (Lords, House of. Gillik vs West Norfolk \& Wisbech Area Health Authority, 1985), establece una jurisprudencia clara al señalar que las normas que permiten que adolescentes dispongan de planificación familiar y/o anticoncepción no vulneran la patria potestad, pues se basan en los principios de interés superior de los niños y las niñas y autonomía progresiva de los mismas/os. Así mismo, el Tribunal Constitucional Chileno en el requerimiento de inconstitucionalidad al D.S. 48 del Ministerio de salud, rol 740-07 del 18 de abril del 2008. Niega la acción de inconstitucionalidad presentada contra la prescripción de Anticoncepción Oral de Emergencia y otros anticonceptivos, y en lo concerniente al consentimiento y confidencialidad de adolescentes para acceder al mismo manifiesta:

(...)El Decreto Supremo N 48, de 2007, del Ministerio de Salud, y las normas contenidas en la sección D bajo el título "Anticoncepción en Población Específica" en el punto 1 "Anticoncepción en Adolescentes", de las "Normas Nacionales sobre Regulación de la Fertilidad", que son parte integrante del mencionado decreto, no vulneran el derecho de los padres a educar a sus hijos ni les impiden el cumplimiento del deber que les incumbe, al establecer la consejería a adolescentes en un marco de confidencialidad sin consentimiento ni conocimiento de los padres. Las normas sobre consejería en condiciones de confidencialidad no impiden, en efecto, a los padres de las adolescentes escoger el establecimiento educativo 
de sus hijas ni transmitir a éstas conocimientos y valores sobre la vida sexual, lo que es suficiente para rechazar el requerimiento en esta parte, sin que dichas normas vulneren el ejercicio legítimo de los derechos de las adolescentes, que también debe ser respetado (...) (Resuelve) Que asimismo se rechaza el requerimiento, en cuanto impugnó la consejería a adolescentes en el marco de confidencialidad sin conocimiento ni consentimiento de los padres (...)

En este mismo sentido, la Guía de supervisión de la salud de las y los adolescentes emitida por el Ministerio de Salud Pública en el 2014 y el Acuerdo Ministerial 2490 que regla la disponibilidad de métodos anticonceptivos en el Sistema Nacional de Salud del Ecuador establece:

Los profesionales de salud saben que ninguna persona requiere autorización de sus familiares o de su pareja para acceder a atención, a anticoncepción, o asesoría y, por lo tanto deben proveer la atención, información y asesoría que requieran (Acuerdo Ministerial No 00002490).

La atención a adolescentes debe garantizar: 6. Su derecho a ser respetado en la evolución de sus facultades, lo que se conoce como autonomía progresiva, que se traduce en la posibilidad de formarse un juicio propio, poder decidir por sí y asumir las consecuencias de su decisión. (Salud en adolescentes. Guía de Supervisión, 2014)

En cuanto al planteamiento de que permitir a adolescentes acceder a servicios de salud sexual, y salud reproductiva sin autorización de sus padres y madres puede ser vulneratorio de otros derechos de sus progenitores como la libertad de conciencia, la libertad 
de educar a sus hijos en sus principios y otros. Es fundamental señalar que garantizar los derechos de los adolescentes no vulnera estos derechos de los padres y madres, pues permitirles acceder a información educación y atención en salud sexual y salud reproductiva no interfiere en la posibilidad de sus progenitores de educarlos conforme a sus valores y creencias. En el supuesto no consentido de que existiera una afectación a los derechos de padres y madres, se debe recordar que por el principio de bienestar superior de niños, niñas y adolescentes, los derechos de estos priman sobre los de cualquier otra persona y se deben tomar las decisiones que sean más favorables a las y los mismos.

Para garantizar que el consentimiento de adolescentes sobre su salud sexual y salud reproductiva resguarde efectivamente sus derechos, es fundamental analizar este tema también desde el principio de autonomía progresiva, pues si bien generalmente el acceso a servicios y su capacidad para tomar decisiones sobre sexualidad y reproducción es un factor protector, pueden haber casos en que se genere una sobrecarga sobre las y los adolescentes al pedirles tomar decisiones para las cuales no tienen la madurez suficiente.

Como no existen pautas claras sobre la edad en que las y los adolescentes pueden consentir, en este artículo esbozare lo que a mi criterio podrían ser tres reglas prácticas en este tema, basadas en el análisis de otras leyes y estándares tanto nacionales como internacionales. Esto sin olvidar que cada caso debe ser analizado de forma particular con el objetivo de garantizar de manera más efectiva los derechos de las y los adolescentes.

La primera regla que en el presente artículo se propone en materia de consentimiento informado para adolescentes, es que a partir de los 16 años, estos pueden tomar cualquier decisión 
sobre su salud sexual y salud reproductiva, incluso cuando se trata del requerimiento de un aborto legal. Esto pues en Ecuador a partir de esta edad se les ha reconocido la autonomía personal y madurez, al permitirles votar, conducir automóviles y ser imputables penalmente, por lo cual en ninguna circunstancia debería restringirse su posibilidad de tomar decisiones sobre su salud, reproducción y su vida sexual. Este planteamiento es coherente con los estándares internacionales en la materia que determinan también a los 16 años como la edad en que estas decisiones en salud no pueden subrogarse (LANSDOWn, Gerison, 2005, La evolución de las facultades del niño, 43).

Esto no implica que adolescentes menores no puedan tomar decisiones sobre su vida sexual y su vida reproductiva, incluyendo la decisión de un aborto legal, caso en que el criterio para tomar la decisión debe ser valorado en base a la madurez de cada adolescente en particular y su bienestar superior (LANSDOWN, Gerison, 22, 43 y 150). Se debe garantizar que el consentimiento prestado por terceros este guiado por el interés superior de las y los adolescentes, entendido como la protección de sus derechos reconocidos en el derecho internacional de los derechos humanos: la salud, la vida, la integridad, la información, la autonomía, la igualdad, la dignidad, los mismos que se constituyen como un límite para cualquier decisión de subrogación. Para analizar la relación entre el bienestar superior de la o el menor con el consentimiento dado o negado por padres y madres, es fundamental valorar también el alcance invasivo de la decisión que se subroga, su impacto para su potencial vida futura y las condiciones en las cuales se realizará la intervención. Por ejemplo, en caso de que la continuación de un embarazo ponga en peligro la salud o la vida de la adolescente, esto debe considerarse como prioritario frente a la opción religiosa de su padre y madre, por lo que en 
caso de que la adolescentes no tenga capacidad probada para consentir, y sus progenitores no consientan, se debe acudir a la Defensoría del Pueblo o a la Junta de Protección de Derechos para solicitar autorización judicial para proceder con la intervención, y precautelar el interés superior de la adolescente.

Al respecto existe un fallo de la Corte Suprema de Estados Unidos en el caso Belloti contra Baird, donde al considerarse que la decisión de una interrupción de un embarazo no es postergable y tiene un fuerte impacto en la vida de las menores, la corte decide que las adolescentes menores no casadas de cualquier edad podían obtener autorización judicial para un aborto sin autorización de su padres o madres, de acuerdo a su interés superior (Corte Suprema de los Estados Unidos, 1979)

Una segunda propuesta es que los y las adolescentes de 14 años o más deberían poder consentir en la generalidad de procedimientos en salud sexual y salud reproductiva, pues de acuerdo al Código Integral Penal, es a partir de esta edad que el consentimiento en menores es válido para aceptar una relación sexual ${ }^{13}$. Lo cual implica que estas y estos adolescentes menores deben contar con toda la información, asesoría y herramientas para tomar decisiones sobre su vida sexual garantizando su bienestar superior y el ejercicio de sus Derechos.

Una tercera propuesta es que a partir de los 11 años, las y los adolescentes deberían recibir información, educación sobre

13 La excepción para la penalización de relaciones con niños, niños y adolescentes menores a 14 años, es que las mismas hayan sido entre niño, niñas o adolescentes menores a 14 años, pues en ese caso no se podría penalizar a ninguna de las partes. 
sexualidad y anticonceptivos sin requerir ninguna autorización. Esto pues a pesar de que la edad de consentimiento legal para aceptar una relación sexual son los 14 años; estudios de comportamiento sexual en adolescentes muestran que una alto porcentaje de la población juvenil menor de 14 años tiene relaciones sexuales consentidas a muy temprana edad con sus pares adolescentes o con personas adultas (CASAS, Lidia, 2005, "Confidencialidad de la información médica, derecho a la salud y consentimiento sexual en adolescentes", 44-46). Esta realidad nos plantea la necesidad de pensar la forma de facilitar el acceso de estas y estos adolescentes a asesoría y atención en salud sexual y salud reproductiva, como una forma de prevenir las infecciones de transmisión sexual, embarazos no deseado, realizar vigilancia sobre situaciones de violencia a las que pueda estar expuestos/ as y crear un ámbito de confianza y seguridad para que él o la adolescente pueda ir tomando decisiones respecto a la propia sexualidad.

Es por esto, que en el marco del Derecho Internacional de Derechos Humanos, muchos países entre ellos Argentina, Chile, Uruguay, y Bolivia, permiten que muchas decisiones en salud sexual y salud reproductiva las tomen adolescentes menores de 14 años. Pues el bien jurídico a proteger es su bienestar, integridad y salud, y la mejor manera de hacerlo es garantizarles condiciones para evitar riesgos y tomar decisiones en situación de información y seguridad. Reconociendo que la vivencia de la sexualidad no reconoce los límites impuestos por las normas sociales y/o jurídicas.

En Ecuador, la legislación y las normas asistenciales constituyen un marco jurídico ideal para garantizar este derecho en adolescentes menores. Es por esto que la regla general para el consentimiento 
en menores de 14 años debe ser apreciar la capacidad para tomar decisiones en cada procedimiento, de acuerdo a su madurez y a su bienestar superior. Ya que la madurez de un/a menor para tomar decisiones sobre su salud debe ser apreciada en función del procedimiento específico, pues la autonomía progresiva tiene diversos grados y un/a menor puede ser maduro para tomar determinadas decisiones en un momento de su vida y no otras, pues no todas las decisiones requieren el mismo nivel de madurez (LANSDOWn, Gerison, 43).

Se debe considerar también que un indicio de madurez es que las y los adolescentes busquen servicios de salud, pues este acto implica conciencia del cuidado de sí mismo y de la responsabilidad frente a sus $\operatorname{actos}^{14}$ (GonzÁlez Vélez, Ana Cristina y Durán, Juanita, 20). Por esto es fundamental que se garantice su bienestar superior que se relaciona con la confidencialidad en salud, pues está comprobado que las y los adolescentes prefieren no asistir a servicios de salud que arriesgarse a que se revele información considerada como delicada por ellos/as a sus padres y madres (MSP, 17-22)

En el Ecuador de acuerdo a las cifras de la Encuesta Nacional de Salud y Nutrición (ENSANUT-2012, 127) únicamente el 79,8\% de adolescentes entre 12 y 14 años conoce sobre anticonceptivos. Esto repercute en la existencia de indicadores (INEC, Anuario de camas y egresos hospitalarios, 313-315) bastante graves en adolescentes menores de 14 años, registrándose sólo en el año

14 Sin embargo, que un adolescente no busque servicios de salud no puede considerarse un indicio de inmadurez, pues la existencia de múltiples y diversas barreras de acceso a los mismos explica fácilmente su desconfianza en el sistema de salud y como la misma los disuade de buscar estos servicios. 
2014, 228 abortos no especificados, 707 partos por cesáreas, y 1243 partos normales, lo cual nos habla de la brecha existente en la asesoría y atención a este grupo de edad.

Para garantizar los derechos de los adolescentes, y su capacidad de consentir es necesario que los profesionales de salud generen las condiciones para el ejercicio de su autonomía, poniendo a disposición de los mismos, explicaciones claras, completas y oportunas; tomando en serio sus dudas, inquietudes y preguntas; no priorizando sus juicios de valor sobre si les parece o no correcto que un/a adolescente haya o piense iniciar su vida sexual; e incentivando su confianza, pues el contexto en que un adolescentes es atendido en el servicio de salud influencia fuertemente su posibilidad de tomar decisiones (R.CoOK, B.M.DicKENS, 19-20).

La madurez de los menores para el ejercicio de su autonomía no es únicamente un asunto del desarrollo cognitivo o moral, sino que en gran medida depende del contexto en el que su madurez es evaluada. Un menor que debe decidir en siendo tratado con hostilidad, bajo la amenaza de que sus comportamientos serán divulgados, evaluado a través de preguntas inquisitivas y prejuiciosas, en espacios que no respeten su privacidad, sin hallar respuestas a sus inquietudes, tiene menos posibilidad de adoptar decisiones maduras y autónomas sobre el ejercicio de sus derechos. Se ha demostrado que los menores tienen una mayor capacidad de demostrar su comprensión de las situaciones en contextos que les resultan familiares, y también que su capacidad de asumir responsabilidades o demostrar su madurez es altamente sensible a la metodología a través de la cual se evalúan 
estas competencias (GonZÁlez Vélez, Ana Cristina y DuRÁn, Juanita, 21)

Por esto la madurez de las y los adolescentes debe considerarse como la posibilidad de construir un dialogo que los empodere y les permita decidir autónomamente. (LANSDOWN, Gerison, 43).

En aquellos casos donde después de haber generado condiciones que promuevan la autonomía de las y los adolescentes, se considere que un menor no tiene madurez suficiente para decidir sobre algún aspecto de su salud sexual y salud reproductiva, en el Ecuador existe un sistema de sustitución de las decisiones y el consentimiento. Este sistema consiste en la asignación de una persona cercana al menor, para subrogar la función de consentir por el o la misma.

En casos de consentimiento subrogado por terceros, se debe garantizar que el mismo este guiado por el interés superior de las y los menores, entendido como la protección de sus derechos reconocidos en el derecho internacional de los derechos humanos: la salud, la vida, la integridad, la información, la autonomía, la igualdad, la dignidad, los mismos que se constituyen como un límite para cualquier decisión de subrogación.

En la implementación práctica del principio de interés superior, es fundamental tomar en cuenta tres premisas fundamentales:

1. La incapacidad de la o el menor no puede convertirse en una desventaja para la protección de su salud y su vida;

2. El ejercicio del derecho a la libertad religiosa de padres y madres, no está sobre el derecho a la vida, salud e integridad de las y los adolescentes y,

3. Nadie puede adoptar decisiones negativas directas que tengan 
como consecuencia el detrimento del bienestar de terceras personas.

Además, cuando se considere que los y las menores no tienen capacidad para toma de decisiones autónomas sobre su salud, esto debe estar argumentado y probado antes de proceder a llamar a una persona adulta para que subrogue las decisiones. De otra manera es ilegal romper la confidencialidad de las y los menores. (SURKUNA, El Secreto Profesional en el ámbito sanitario)

En cualquier caso se requiere el asentimiento informado de las y los menores sobre los procedimientos, que implica que los mismos deben ser escuchados y preguntados sobre el procedimiento y que sus opiniones deben ser una guía para la toma de decisiones y una base para la evaluación de la relación entre bienestar superior de la o el menor con el consentimiento dado o negado por padres y madres frente a un determinado procedimiento (GARcíA, Yolanda, 2006, "Salud, autonomia y factor religioso: Una compleja encrucijada en el supuesto de los menores", 27).

Para analizar la relación entre el bienestar superior de la o el menor con el consentimiento dado o negado por padres y madres, es fundamental valorar también el alcance invasivo de la decisión que se subroga, su impacto para su potencial vida futura y las condiciones en las cuales se realizará la intervención.

Cuando un menor carece de capacidad para decidir directamente en asuntos relativos a la salud, el principio rector que sustituye al de autonomía es la búsqueda de su bienestar y salud. (...) De allí que se prohíben las decisiones que afectan la salud, integridad física, vida o libertades de los menores, entre muchos otros 
derechos. Las decisiones que se adoptan en el ejercicio de los derechos sexuales y reproductivos de los menores deben proteger siempre su derecho a la salud sexual y reproductiva, y su salud más ampliamente (GonZÁLEZ VéLEZ, Ana Cristina y DuRÁn, Juanita, 25)

Como ya lo hemos enunciado, en el caso de adolescentes es fundamental el abordaje integral por parte de los profesionales de salud, pues el componente informativo del consentimiento requiere la constatación de la comprensión de la información y la evaluación del tipo de información y la cantidad de la misma que se suministra.
.(...) los profesionales de la salud tienen un papel activo en el suministro de información para el consentimiento en el caso de menores, orientado a garantizar que quien decide este efectivamente informado y ejerce su derecho de manera autónoma (GonzÁlez VÉlez, Ana Cristina y DuRÁn, Juanita, 27).

El contenido de la información que se entrega a adolescentes, previo su consentimiento informado no debe diferenciarse del suministrado a adultos. Sin embargo, como lo hemos mencionado reiteradas veces es crucial la forma como se ofrece esta información, con el objetivo de generar condiciones para el empoderamiento de los y las adolescentes.

Por lo señalado, se recomienda que se considere que un/a adolescente puede ser más vulnerable a los juicios de valor 
negativos, amenazantes o peyorativo; la confidencialidad ${ }^{15}$ en salud debe asegurarse incluso frente a sus padres y madres;16 y que es fundamental que la información sea lo más completa posible, y que se garantice que la misma este basada en evidencia científica (González Vélez, Ana Cristina y Durán, Juanita, 28) Es importante también conocer que no prestar los servicios que los menores con capacidad para decidir solicitan y requieren, vulnera sus derechos y los principios bioéticos en la atención sanitaria. Por lo que una intervención adecuada o la usencia de intervención con el argumento de falta de representante legal, genera responsabilidad legal para los profesionales de salud.

\section{CONCLUSIONES}

De acuerdo a la Constitución Ecuatoriana, Convención sobre los Derechos del niño, y el Código Orgánico de la Niñez y la Adolescencia, niños, niñas y adolescentes son titulares de derechos, y de acuerdo a sus capacidades evolutivas van ganando autonomía progresiva para la toma de decisiones sobre sus vidas. Esto implica la existencia de un equilibrio entre el reconocimiento de las niñas, niños y adolescentes como agentes activos en sus propias vidas que

15 Existe evidencia de que la mayoría de las menores que buscan servicios de salud sexual y reproductiva no lo haría si se informara a sus padres que usan anticoncepción, aunque solo el 1\% estaría dispuesto a dejar de tener relaciones sexuales y de que los abortos tardíos en adolescentes se deben al miedo de decirles a sus padres sobre el embarazo. (Planned Parenthood Federation of America, 2004)

16 La importancia de la confidencialidad se acentúa cuando se trata de los padres, pues los comportamientos en materia sexual y reproductiva de las/los menores pueden ser abiertamente contrarios a las creencias de estos, quienes además. pueden estar interesados en disuadir, u oponerse cuando las normas jurídicas así lo permiten, a que las/los menores adopten determinadas decisiones. 
tienen derecho a ser respetados como ciudadanos, como personas y como titulares de derechos con una autonomía creciente, y el derecho que tienen a diferentes niveles de protección de acuerdo a su grado de vulnerabilidad, constituyéndose la edad en un factor que protección y no de vulnerabilidad. (Convención sobre los Derechos de los niños, 1989)

El principio de bienestar superior del niño, niña y adolescentes reconocido en la Constitución del Ecuador, en la Convención de los Derechos del Niño y en el Código Orgánico de la Niñez y la Adolescencia, establece que en cualquier caso la titularidad de derechos de los niños, niñas y adolescentes se mantiene sobre las limitaciones que pueden establecerse al ejercicio de los mismos. Las y los adolescentes tienen derecho a ejercer sus derechos sexuales y derechos reproductivos, incluyendo su derecho a acceder a anticoncepción, educación sexual integral, atención en salud sexual y salud reproductiva, en tanto los mismos constituyen medios de protección de sus derechos a la salud, la educación y la vida (CoOK, R, 20-21). Constituyendo cualquier intento de restringir este acceso a información, educación y prestaciones de salud una injerencia arbitraria en su vida privada y un ejercicio desproporcionado de su autoridad.

En ningún caso, se debe permitir que se subrogue consentimiento por causas de edad, en mayores de 16 años. Pues la mayoría de legislaciones consideran que un adolescente a partir de los 16 años puede tomar decisiones sobre su salud y vida sin ningún problema.Además a esta edad generalmente las y los adolescentes pueden conducir, votar, y ser imputados penalmente, lo cual demuestra que se les reconoce madurez suficiente para tomar decisiones sobre su salud, y mucho más aquellas referidas a su sexualidad y reproducción. 
A partir de los 14 años, en Ecuador se reconoce el consentimiento de adolescentes para tener relaciones sexuales. Razón por lo que desde antes de esta edad, se les debe garantizar las condiciones para tomar estas decisiones de forma segura y sin riesgos, para lo cual es fundamental que se garantice su atención e información en salud con confidencialidad y que se les permita tomar decisiones informadas sobre su sexualidad y reproducción.

En el caso de adolescentes menores de 14 años, es fundamental considerar que ellas y ellos están teniendo relaciones sexuales en la actualidad. Por lo cual asegurar su acceso a atención e información en salud sexual y salud reproductiva es fundamental para garantizar su bienestar superior y prevenir riesgos. En este caso se debe valorar individualmente la capacidad de cada adolescente menor para tomar decisiones referentes a salud sexual y salud reproductiva, en relación a cada proceso específico. En casos de información, asesoría y acceso a anticoncepción y anticoncepción oral de emergencia, las y los menores de 14 años pueden consentir libre y voluntariamente sobre los mismos sin la necesidad de un adulto de acuerdo a la normativa interna y los principios del Derechos Internacional de Derechos Humanos. 


\section{BIBLIOGRAFÍA}

BELLOTI contra Baird, 443 US 622 (Corte Suprema de los Estados Unidos 1979).

CONVENCIÓN sobre los Derechos de los niños. (1989).

AGUILAR CARVALLO, G. (2008). "El principio del interés superior del niño y la Corte Interamericana de Derechos Humanos". Estudios Constitucionales. Año 6 Vol I, 223-247.

CASAS, L. (2005). Confidencialidad de la información médica, derecho a la salud y consentimiento sexual en adolescentes. Revista de la Sociedad Chilena de Obstetricia y Ginecología Infantil y de la Adolescencia.

CEPAL. (2014). Panorama Social de Latino América.

CHRISTOFIDES. (2014). Risk factors for unplanned and unwanted teenage pregnancies occurring over two years of followup among a cohort of young South African women.

CONGRESO NACIONAL DEL ECUADOR. (2006). Ley Orgánica de Salud. Quito : Registro Oficial.

COnstituyente, A. N. (2008). Constitución de la República del Ecuador. 
COOK, R. A. (2005). Adolescents and consent to treatment. Journal of Gynecology and Obstetrics, pp. 179-184.

FREIRE WB, R.M. (2013). Encuesta nacional de salud y nutrición 2011-2012. INEC.

FUNDACIÓN Desafio. (2015). Vidas Robadas: entre la omisión y la premeditación. Quito.

GARCÍA, D., Jarobo, Y., Espíldora, N. M., \& Ríos, J. (2001). Toma de decisiones en el paciente menor de edad. Instituto de Bioética. Fundación Ciencias de la Salud.

GARCíA, F. J. (2014). Tratados Internacionales de Derechos Humanos: Diferenciación con otros tratados. Derechos Ecuador, http://www.derechoecuador. com/articulos/detalle/archive/doctrinas/ derechoconstitucional/2014/01/23/ tratados internacionales -de-derechos-humanos-diferenciacion-con-otros-tratados.

GARCíA, Y. (2006). Salud, autonomía y factor religioso: Una compleja encrucijada en el supuesto de los menores. La salud: Intimidad y libertades informativas, 11-40.

GONZÁLEZ VÉLEZ,A.C., \& Durán, J. (2010). Derechos sexuales $\mathrm{y}$ reproductivos de la gente joven.

HERNÁNDEZ, Mónica. (2014). Versión Borrador Plan Nacional de Fortalecimiento de la Familia.

INEC. (2010). CENSO. 
JIMBO, R. (2014). Educación sexual versus abstinencia en adolescentes. Quito: MSP.

LANSDOWN, G. (2005). La evaluación de las facultades del niño. Florencia: Centro de Investigaciones Innocenti de UNICEF.

LORDS, H. o. (1985). Gillik vs West Norfolk \& Wisbech Area Health Authority. Reino Unido.

MADDALENO, M. P. (2003). Salud y desarrollo de adolescentesy jóvenes en Latinoamerica y El Caribe: desafios para la proxima decada. Revista Salud Publica de Mexico vol. 45.

MINISTERIO DE SALUD PUBLICA. (2014). Salud en adolescentes. Guía de Supervisión. Obtenido de Disponible en: http:// salud.gob.ec

MSP. (2011). Datos esenciales de salud: una mirada a la década 2000-2010.

MSP. (2014). Salud en adolescentes. Guía de Supervisión. Obtenido de Disponible en: http://salud.gob.ec

MSP Y CONASA. (2007). Politica de Salud y Derechos Sexuales y Reproductivos.

NACIONAL, C. (2003). Código de la Niñez y la adolescencia.

NACIONES UNIDAS.(1995). Informe de la Conferencia Internacional sobre la Población y Desarrollo. Nueva York. 
NACIONES UNIDAS. (1996). Informe de la Cuarta Conferencia Mundial sobre la mujer. Nueva York.

OIJ. (2014). Invertir para transformar: la juventud como protagonista del Desarrollo.

PACHECO, V. M. (2015). Consentimiento Informado (fundado).

PLANNED PARENTHOOD FEDERATION OF AMERICA. (2004). Adolescentes, aborto y leres de instrusion del gobierno. New York: Biblioteca Katherine Dexter Mc Cormic.

R. COOK, B. (2009). El reconocimiento de "la evolución de las facultades" de los adolescentes para tomar decisiones en cuanto a su salud reproductiva.

SAVE THE CHILDREM. (2003). Diagnóstico sobre la situacion de niños, niñas y adolescentes en 21 paises de latinoamerica.

SIMON, F. (2008). Derechos de la Niñez y Adolescencia: De la Convención sobre los del Niño a las Legislaciones Integrales. Quito: Juridica Cevallos.

, (2010). Garantías de los derechos de la infancia y adolescencia . En R. y. Ávila, Derechos y garantías de la niñez y adolescencia: Hacia la consolidación de la doctrina de protección integral (pp. 441-484). Quito.

TRIVEDI D, B. F. (2007). Update on review of reviews on teenage pregnancy and parenthood.Centre for Research in Primary and Community Care University of Hertfordshire. 
UNESCO. (2010). Orientaciones Técnicas Internacionales sobre Educación en Sexualidad. Santiago de Chile.

UNFPA. (2014). Estado de la población Mundial: El poder de 1,800 millones: Adolescentes, jóvenes y la transformación del futuro.

UNFPA, Equipo de Apoyo Técnico de América Latina y el Caribe. (s/f). Guía de argumentos para la promoción y defensa de los compromisos de la conferencia internacional de población y desarrollo. México.

UNICEF. (2011). Estado Mundial de la Infancia 2011: La adolescencia una etapa de oportunidades.

UNICEF y Plan Internacional. (2014). Vivencias y relatos sobre el embarazo en adolescentes. Una aproximación a los factores culturales, sociales y emocionales a partir de un estudio en seis paises de la region. Informe Final. Panamá.

VALENZUELA,L.C.(2010).La regulación legal sobre la capacidad de los adolescentes en algunos países de América Latina y su impacto en la salud sexual y reproductiva.

WELLER SC, D.-B. K. (2001). Condom effectiveness in reducing heterosexual HIV transmission: Review.

WHO. (2007). Adolescent pregnancy -Unmet needs and undone deeds. A review of the literature and programes. 